\title{
Isoform and protein region abnormalities of dysbindin and copper transporter proteins in
} postmortem schizophrenia substantia nigra.

Running Title: Pathology of dysbindin and copper in schizophrenia

Kirsten E. Schoonover ${ }^{1}$, Rosalinda C. Roberts ${ }^{2}$

${ }^{1}$ Department of Psychology and Behavioral Neuroscience, ${ }^{2}$ Department of Psychiatry and

Behavioral Neurobiology, University of Alabama at Birmingham, Birmingham, AL 35294

Corresponding author information:

12 Kirsten Schoonover, B.A.

13 Sparks Center 866

$1417202^{\text {nd }}$ Ave South

Birmingham, AL 35294

Tel. 304-437-4554

17 Fax. 205-996-9377

18 Kschoon1@uab.edu

Abstract: 200

Text Body: 4,176

Tables: 1

Figures: 4

28

29 
Abstract

Objective: Dysbindin is downregulated in several schizophrenia brain regions and modulates copper transport required for myelination and monoamine metabolism. We sought to determine dysbindin and copper transporter protein expression in schizophrenia subjects. Methods: We studied the substantia nigra (which exhibits one of the highest copper contents of the human brain) using Western blot analysis. We characterized specific protein domains of copper transporters ATP7A, CTR1, ATP7B, and dysbindin isoforms 1A and 1B/C in postmortem substantia nigra in schizophrenia subjects $(n=15)$ and matched controls $(n=11)$. As a preliminary investigation, we examined medication status in medicated $(n=11)$ versus unmedicated schizophrenia subjects $(n=4)$.

Results: The combined schizophrenia group exhibited increased levels of C-terminus, but not N-terminus, ATP7A. Schizophrenia subjects expressed less transmembrane CTR1 and dysbindin $1 \mathrm{~B} / \mathrm{C}$ than controls. When subdivided, the increased C-terminus ATP7A protein was present only in medicated subjects versus controls. Unmedicated subjects exhibited less Nterminus ATP7A protein than controls and medicated subjects, suggesting medication-induced rescue of the ATP7A N-terminus. Transmembrane CTR1 was decreased to a similar extent in both treatment groups versus controls, suggesting no medication effect.

Conclusions: These results provide the first evidence of disrupted copper transport into and within schizophrenia nigral cells that may be modulated by specific dysbindin isoforms and antipsychotic treatment.

Keywords: schizophrenia, dysbindin, copper, substantia nigra, postmortem 
Introduction

Schizophrenia manifests in early adulthood with cognitive, positive, and negative

72 symptoms. Several genes have been associated with schizophrenia risk (Schizophrenia Working

73 Group of the Psychiatric Genomics Consortium 2014). The dystrobrevin binding protein 1

74 (DTNBP1) gene encodes the dysbindin protein family, dysbindin-1 (Talbot 2009; Talbot et al.

75 2009), and is a top candidate gene for schizophrenia (Straub et al. 2002). Allelic variation of

76 DTNBP1 was associated with schizophrenia shortly after its discovery (Straub et al. 2002), and

77 has been associated with dysbindin protein decreases in cortex, midbrain, and hippocampus in

78 schizophrenia (Talbot 2009; Tang et al. 2009; Weickert et al. 2008; Weickert et al. 2004).

$79 D T N B P 1$ variations have been associated with impaired white matter integrity in healthy adults

80 (Nickl-Jockschat et al. 2012), loss of grey matter volume in preteenagers (Tognin et al. 2011),

81 abnormalities in neurite outgrowth and morphology (Dickman and Davis 2009), as well as

82 decreased cognitive functions (Burdick et al. 2006), including working, visual, and verbal

83 memory deficits (Varela-Gomez et al. 2015). Additionally, schizophrenia subjects show

84 decreases in specific dysbindin isoforms in a region specific manner, especially in brain regions

85 involved in cognition (e.g., prefrontal cortex and hippocampus) Tang et al. 2009; Weickert et al.

86 2008; Talbot et al. 2004). Together, these data suggest that dysbindin may be involved in

87 schizophrenia cognitive symptomology, but the mechanism remains unknown.

88 In mice, dysbindin knockout results in spatial (Cox et al. 2009) and working memory

89 deficits (Papaleo et al. 2012), and increased compulsive and impulsive behaviors (Carr et al.

90 2013), not unlike the cognitive impairments exhibited by schizophrenia patients with disease-

91 related DTNBP1 variants (Burdick et al. 2006; Varela-Gomez et al. 2015). Additionally,

92 dysfunctional dysbindin in animal models results in a particularly intriguing consequence of 
interest: a decrease in copper transporters ATP7A and CTR1 (Gokhale et al. 2015). Together

94 ATP7A and CTR1 facilitate copper transport between the blood and the brain (Figure 1A), as

95 well as intracellular transport.

Copper plays a key role in development and homeostatic function and is crucial for many

97 cellular functions including monoamine metabolism, mitochondrial activity, and myelination

98 (Sato et al. 1994). While copper and its enzymes are found outside of the brain, we will focus on

99 brain. Copper dysfunction results in Wilson's or Menkes disease, characterized by copper

toxicity or deficiency, respectively (Wilson 1934; Menkes et al. 1962). Cellular copper is highly

regulated, as free copper can induce oxidative stress and cellular damage (Halliwell and

102 Gutteridge 2007). During normal function, copper is taken from the bloodstream across the

103 blood brain barrier (BBB) into astrocytes and then neurons via CTR1 at the plasma membrane

104 (Scheiber et al. 2010). Once inside the cell, copper is bound by metallochaperones (Maryon et al.

105 2013) and delivered to the trans-Golgi network (TGN). ATP7A is located within the TGN

106 (Yamaguchi et al. 1996) and distributes copper to metallochaperones (e.g., SCO1) which

107 transport the copper to their needed location within the cell (e.g., mitochondria)(Davies et al.

108 2013; Leary et al. 2007). CTR1 knockdown and/or loss results in developmental defects and

109 lethality (Lee et al. 2001), and total loss of ATP7A results in Menkes disease and lethality

110 (Menkes et al. 1962), exemplifying the incredible importance of these transporters in

111 homeostasis and function.

Interestingly, decreasing copper activity through inhibited transporters or administering

113 the copper chelator cuprizone to mice produces demyelination, altered neurotransmitters, and

114 decreased oligodendrocytic protein expression (Gokhale et al. 2015; Gregg et al. 2009; Herring

115 and Konradi 2011). Additionally, reduced copper activity results in schizophrenia-like behavioral 
116 impairments, such as deficits in novel object recognition, spatial memory tasks, pre-pulse

117 inhibition, social interaction, and anxiety (Talbot et al. 2009; Gregg et al. 2009; Herring and

118 Konradi 2011). The role of copper in schizophrenia has been rarely studied, but increased copper

119 in the blood of schizophrenia subjects has been observed in many, but not all studies (see

120 Vidovic et al. 2013 and references therein). However, the cause of this copper increase or

121 resulting functional consequences have not been studied. We hypothesize that schizophrenia

122 subjects exhibit excess blood copper due to faulty copper transport across the blood brain barrier

$123(\mathrm{BBB})$

124 Importantly, several intracellular components that interact with copper are reported to be

125 abnormal in schizophrenia (Figure 1B). For example, schizophrenia subjects exhibit a deficit of

126 SCO1, the metallochaperone responsible for delivering copper to the catalytic core of

127 cytochrome C oxidase (COX) required for ATP synthesis (Leary et al. 2007; Purcell et al. 2014).

128 Not only do schizophrenia subjects exhibit less COX protein, they also exhibit decreased ATP

129 (Volz et al. 2000; Cavelier et al. 1995). Additionally, schizophrenia subjects exhibit decreased

130 metallothionein and glutiothione, responsible for intracellular copper chaperoning and transport,

131 that are rescued with antipsychotic treatment (Xuan et al. 2015; Do et al. 2000)(Figure 1B).

132 While these deficits are well replicated, they have not been studied in the context of copper.

133 Given that these mechanisms require copper, and that schizophrenia subjects exhibit alterations

134 of intracellular copper pathway proteins (Figure 1B), copper may play an important role in

135 schizophrenia pathology. Without proper regulation of the copper system and its transporters

136 CTR1 and ATP7A, a cascade of schizophrenia-like effects can occur such as altered cellular

137 energy metabolism (Leary et al. 2007; Volz et al. 2000; Cavelier et al. 1995), demyelination 
(Xuan et al. 2015), oxidative stress (Do et al. 2000), or even cellular death (Menkes et al. 1962;

139 Halliwell and Gutteridge 2007; Lee et al. 2001).

141 combined pathology in schizophrenia. The substantia nigra (SN) exhibits one of the highest

142 levels of copper within the brain and the highest level of the copper chaperone Atox1 (Davies et

143 al. 2013), indicating the SN has a high demand for cellular copper. Therefore, we measured 1)

144 dysbindin isoforms $1 \mathrm{~A}$ and $1 \mathrm{~B} / \mathrm{C}$, encoded by risk factor gene $D T N B P 1 ; 2)$ copper transporter

145 CTR1, responsible for copper transport across the BBB; 3) copper-transporting P-type ATPase,

146 ATP7A, which works in conjunction with CTR1 for intracellular copper transport; and ATP7A

147 homolog ATP7B, responsible for transfer of copper to the secretory pathway. We also conducted

148 a preliminary analysis of medication status, as there are biological correlates to medication

149 status. Our hypothesis is illustrated in Figure 1A. We hypothesize schizophrenia subjects exhibit

150 deficits in copper transport in a copper-rich brain region potentially in relation to dysbindin

151 alterations (Figure 1B). This work has been presented in preliminary form (Schoonover and

152 Roberts 2016). 
Postmortem Brains Birmingham. The schizophrenia cohort was the same as previously studied (Schoonover et al. 2017). Schizophrenia cases $(n=15)$ were matched and compared to normal controls $(N C, n=11)$.

161 As a preliminary investigation, the schizophrenia group was subdivided by treatment status: off

162 medication (SZ-Off, $\mathrm{n}=4$ ) or on medication (SZ-On, $\mathrm{n}=11$ ). Diagnosis of schizophrenia was

163 confirmed independently by two psychiatrists based on DSM criteria at the time of diagnosis

164 (DSM-III-R through DSM-IV-TR) using the Structured Clinical Interview for the DSM (SCID).

165 Subject clinical information (such as age of disease onset, symptomology, and treatment status)

166 was obtained from autopsy and medical records, and family interviews. Placement into the off-

167 medication schizophrenia group required being unmedicated for at least six months prior to

168 death. Cases were selected based on the best match of demographic factors age, race, sex,

169 postmortem interval (PMI), sample $\mathrm{pH}$, and number of years frozen (Table 1A). Exclusionary

170 criteria for this cohort were: history/evidence of intravenous drug abuse, HIV/AIDS, Hepatitis B,

171 head trauma, comorbid neurological disorders, custodial death, fire victims, unknown next of

172 kin, children, or decomposed subjects. In addition, comorbid mental illness in schizophrenia

173 subjects and history of serious mental illness for NCs were exclusion factors. Agonal status has

174 not been shown to have any effect on protein (Stan et al. 2006), and therefore is not an issue with

175 the current investigation. 
Western Blotting

Tissue and Protein preparation

The SN was blocked as done previously (Schoonover et al. 2017). In SN coronal sections, caudal sections were caudal to third nerve rootlets. The blocks were trimmed to remove non-nigral tissue. A perimeter of approximately $2 \mathrm{~mm}$ of non-nigral tissue remained. Frozen caudal SN was sonicated in lysis buffer (500ul/0.1g of human tissue) containing Tris-HCL (pH 8.0), EDTA, sodium chloride, sodium dodecyl sulfate, and a protease inhibitor cocktail (Sigma; P8340). Tissue homogenate was centrifuged at $13,500 \mathrm{rpm}$ for $15 \mathrm{mins}$ at $4^{\circ} \mathrm{C}$. Supernatant (total cell lysate) was then extracted and protein concentration determined via the Lowry method (BioRad, Hercules, CA, USA; 500-0113, 500-0114).

Gel electrophoresis and western blotting

Western blots were used to measure N- and C-terminal ATP7A, ATP7B, extracellular and transmembrane CTR1, and dysbindin 1A and 1B/C protein levels and performed as previously reported (Schoonover et al. 2017), with the following exceptions. Samples intended

191 for N-terminal ATP7A assays were not heated to avoid protein aggregation. Samples intended

192 for all other assays were heated to $95^{\circ} \mathrm{C}$ for 5 mins. Protein extracts $(60 \mu \mathrm{g})$ were loaded onto $4 \%$ -

$19320 \%$ gradient polyacrylamide gels (Lonza, Basel, Switzerland; 58505). Proteins were resolved by 194 sodium dodecyl sulfate-polyacrylamide gel electrophoresis at $150 \mathrm{~V}$ for $1 \mathrm{hr} 15 \mathrm{mins}$, and then 195 transferred at 30V for $21 \mathrm{hrs}$ onto polyvinylidene fluoride (PVDF) membranes (Bio-Rad,

196 Hercules, CA, USA; 162-0174) at $4^{\circ} \mathrm{C}$. Representative western blots are shown in Figure 2A; full 197 blots are shown in Figure S1. 
concentrations were used: rabbit anti-N-terminal ATP7A, 1:500, Novus Biologicals (NBP154906); rabbit anti-C-terminal ATP7A, 1:2000, Aviva Systems Biology (ARP33798_P050); rabbit anti-ATP7B, 1:1000, Abcam (ab135571); rabbit anti-transmembrane CTR1, 1:2,000, Novus Biologicals (NB100-402); rabbit anti-extracellular CTR1, 1:1000, Aviva Systems Biology (ARP43824_P050); rabbit anti-Dysbindin (targeting 1A and 1B/C), 1:2,000, Abcam (ab133652); and mouse anti-actin, 1:40,000, Millipore (MAB1501). Each blot contained a mixture of NC and schizophrenia subjects, and was performed in duplicate. The membranes were blocked for $1 \mathrm{hr}$ in 5\% milk in Tris-Buffered Saline with Tween 20 (TBST). Antigen presence was detected by

208 incubating the primary antibody with the PVDF membrane $21 \mathrm{hrs}$ at $4^{\circ} \mathrm{C}$. The bands were

209 visualized using chemiluminescence (Bio-Rad; 170-5018), exposing Sigma-Aldrich Carestream

210 Kodak BioMax XAR films (166-0760). conducted a preadsorption experiment for $\mathrm{N}$ - and C-terminal ATP7A, and extracellular and

214 transmembrane CTR1. Two identical blots containing samples from two separate controls were

215 used. One blot was incubated with primary antibody; the second blot was incubated with a 216 preadsorbed antibody mixture comprised of $1 \%$ milk containing primary antibody (the same

217 concentration as used above) that incubated for 30 minutes at room temperature with the 218 corresponding blocking peptide (1:500 concentration). The following blocking peptides were 219 used: N-terminus ATP7A, Novus Biologicals (NBP1-54906PEP); C-terminus ATP7A, Aviva 220 Systems Biology (AAP33798); transmembrane CTR1, Novus Biologicals (NB100-402PEP); and

221 extracellular CTR1, Aviva Systems Biology (AAP43824). Verifying ATP7A and CTR1 antibody 222 specificity with knockout animal models is not possible due to embryonic lethality (Menkes et al. 
223 1962; Hua et al. 2010). Dysbindin knockout models are viable and well-characterized (Talbot

224 2009)- additionally, it was measured at its expected molecular weight in the present study.

226 Analyses

227 Data

As described previously (Schoonover et al. 2017), films were scanned at 600 dpi using a

231 Industries Inc.; Mishawaka, IN, USA; T2120, series \#130501) that each measurement was

232 calibrated to. ImageJ was used to perform a background subtraction for each film. All optical 233 density values for each protein were normalized to actin, and then to the averaged NC. These 234 values were averaged for duplicate samples.

Statistics

237 Demographics and tissue quality were tested using ANOVA and/or t-tests. Categorical variables

238 were assessed using a chi-square test. Outliers were detected using ROUT $(\mathrm{Q}=1.0 \%)$ and

239 Grubb's method (alpha=0.05) via Prism 7. Only one schizophrenia ATP7A value qualified for

240 removal for the schizophrenia/normal control analysis. Two schizophrenia subjects had CTR1

241 values that qualified for removal for the schizophrenia/normal control analysis and the treatment

242 status analysis. The data were assessed for normality with both a Shapiro-Wilk test and a

243 D'Agostini and Pearson test. If both tests revealed normally distributed data, parametric tests

244 were used (two groups, unpaired t-test; three groups, one-way ANOVA). If not, non-parametric

245 tests were used (two groups, Mann-Whitney U test; three groups, Kruskal-Wallis H test). 
246 Significant omnibus tests were followed by planned, uncorrected comparisons suggested by

247 Prism 7: parametric tests were followed by an uncorrected Fisher's LSD test, while

248 nonparametric tests were followed by an uncorrected Dunn's comparison. Furthermore, Brown-

249 Forsythe and Bartlett's tests were used to compare standard deviations. Correlational analyses

250 were performed between results and PMI, age, years frozen, and $\mathrm{pH}$ to elucidate potential

251 relationships among these variables. Based on these results, years frozen was used as a covariate

252 in the dysbindin $1 \mathrm{~B} / \mathrm{C}$ analysis. 
Results

254 Demographics

The control (NC) and schizophrenia groups (as a whole and when subdivided by treatment status) were well matched for demographic and tissue quality variables and did not significantly differ (Table 1A). Correlation analysis between protein concentrations and demographic variables only showed a significant between-group difference for dysbindin 1B/C and years frozen (Table 1B). Thus, years frozen was used as a covariate in the dysbindin 1B/C

260 analysis.

All proteins, with the exception of C-terminus ATP7A and transmembrane CTR1, were measured at their expected molecular weights and were successfully blocked by their respective

265 blocking peptide (Figure 2A,B; for full blots see Supplementary Figure S1). While not at their expected molecular weight, the measured C-terminus ATP7A and transmembrane CTR1 bands

267 were verified with blocking peptide.

269 schizophrenia subjects exhibited significantly more C-terminus ATP7A protein than controls

$270 \quad(\mathrm{p}=0.005$; Figure 3A). However, when subdivided by medication status, the N-terminus protein

271 levels were significantly lower in unmedicated subjects versus medicated subjects $(\mathrm{p}=0.007)$ and

272 controls ( $\mathrm{p}=0.017$; Figure 3B). Analysis of treatment status revealed that C-terminal ATP7A

273 protein levels were significantly increased only in medicated subjects $(\mathrm{p}=0.013$; Figure $3 \mathrm{~B})$. 
277 CTR1 was decreased by a similar amount in both medicated $(\mathrm{p}=0.007)$ and unmedicated

278 schizophrenia patients $(\mathrm{p}=0.001$; Figure $3 \mathrm{D})$, and there was no effect of medication on the

279 extracellular CTR1 levels.

280 Dysbindin 1A protein levels were not altered in the whole schizophrenia group or when

281 divided by treatment status (Figure 3E, 3F). Decreased dysbindin 1B/C was observed in

282 schizophrenia subjects versus controls ( $\mathrm{p}=0.046$; Figure $3 \mathrm{E})$, but no effects of antipsychotics

283 were observed (Figure 3F). However, the significant effect was reduced to a trend when the

284 variable years frozen was implemented as a covariate $(\mathrm{p}=0.062)$.

285 No changes of ATP7B were observed in the whole schizophrenia group or when

286 subdivided by treatment status (Figure 3G; Figure 3H).

287 Schizophrenia subjects treated with antipsychotic medication were further divided by

288 antipsychotic type (typical versus atypical antipsychotics). No differences were found (data not 289 shown). 
Discussion

This is the first study to investigate dysbindin and copper transporters as a combined

293

294

295

296

297

298

299

300

301

302

303

304

305

306

307

308

309

310

311

312

313

Limitations

Our sample size was small and as is typical for postmortem investigations, none of our subjects were first-episode and/or antipsychotic-naïve (Schoonover et al. 2017; Howes et al. 2013). Medication history could affect our results, so we divided the schizophrenia group by treatment status and type to eliminate this confounding variable. However, there were only four unmedicated cases, and therefore analysis of treatment status can only be considered preliminary. No differences were found between schizophrenia subjects treated with typical versus atypical antipsychotics (data not shown).

\section{Isoform-Specific Dysbindin Alterations}

Given the association between dysbindin allelic variations and protein expression in schizophrenia (Talbot 2009; Straub et al. 2002; Tang et al. 2009; Weickert et al. 2004, 2008), we anticipated decreased dysbindin expression in schizophrenia. Interestingly, schizophrenia subjects exhibited decreased dysbindin 1B/C, but not isoform 1A. Dysbindin 1 has three isoforms known as dysbindin 1A, 1B, and 1C (Talbot et al. 2009; Talbot et al. 2011). Dysbindin $1 \mathrm{~A}$ is found in the post-synaptic density and thought to be involved in dendritic spine 
314 homeostasis, while dysbindin 1B is found exclusively in synaptic vesicles in the presynaptic

315 axon terminal (Talbot et al. 2011), and is involved in glutamatergic transmission (Numakawa et

316 al. 2004). Dysbindin 1C is found in both places, but primarily is located in the post-synaptic

317 density and involved in dendritic spine function (Talbot et al. 2011). The substantia nigra is

318 relatively spine free (Schwyn and Fox 1974) and the majority of synapses are GABAergic

319 (Tepper and Lee 2007). Approximately 30\% of inputs to the SN are glutamatergic (Smith et al.

320 1996, Parent et al. 1999). Our findings of modest decreases in the dysbindin 1B/C isoform

321 implicate abnormalities in presynaptic glutamate terminals, which can lead to impaired glutamate

322 transmission (Numakawa et al. 2004). Since neurons in the substantia nigra are mostly aspiny,

323 the isoforms $1 \mathrm{~A}$ and $1 \mathrm{C}$, which are involved in spine function, may have little consequence in

324 this brain region.

Other groups have also found decreases in specific isoforms in schizophrenia in various

326 brain regions, such as superior temporal gyrus (1A), dorsolateral prefrontal cortex (1C), and

327 hippocampus (1B/C) (Tang et al. 2009; Talbot et al. 2011). These results, taken together, indicate

328 isoform-specific roles of dysbindin in schizophrenia that are brain region-specific. Given that

329 dysbindin isoforms are differentially associated with synaptic function (Talbot et al. 2009;

330 Numakawa et al. 2004), which is repeatedly found to be abnormal in schizophrenia (Faludi and

331 Mirnics 2011; Roberts et al. 2012), isoform-specific changes observed in the present study are

332 consistent with previous findings.

334 Copper Transporter Deficits

Based on a report of decreased copper transporters in dysbindin knockout mice (Gokhale

336 et al. 2015), we hypothesized dysregulated copper transporters CTR1 and ATP7A as a 
consequence of downregulated dysbindin in schizophrenia subjects. While our results support our hypothesis, we did not anticipate the protein-region specific alterations we observed. We observed a surplus of C-, but not N-terminus, ATP7A in the whole schizophrenia group. The surplus of C terminal ATP7A was significant only in medicated subjects; however, there was no difference between medicated and unmedicated subjects, suggesting no effect of antipsychotic

342 drugs. However, analysis of treatment status revealed what may be an inherent deficit of Nterminal ATP7A in schizophrenia that is rectified with antipsychotic treatment.

345 was measured at its expected molecular weight), it potentially highlights a recurring and well-

346 established alteration of protein phosphorylation in schizophrenia. For example, dysregulated

347 kinase activity has been observed (Banerjee et al. 2015; Singh 2013), affecting, for example, 348 phosphorylation of MAP2 and NMDAR proteins (Shelton et al. 2015; Banerjee et al. 2015;

349 Ramkumar et al. 2018). The antibody used to assess N-terminal ATP7A does not recognize 350 phosphorylated ATP7A protein. Therefore, perhaps the decreased N-terminal ATP7A observed

351 in unmedicated schizophrenia subjects does not represent a loss of protein, but rather post-

352 translational modifications that alter the phosphorylation state. In fact, upon the binding of 353 copper to one of the six metal binding domains (Figure 4A) of N-terminal ATP7A, $\gamma$-phosphate

354 is transferred from ATP to the Asp residue within the P domain of the protein (see Figure 4 in

355 Lutsenko et al. 2007; Valerde et al. 2008). This results in transient phosphorylation that is

356 reversed (dephosphorylated) upon the release of copper into the lumen (Voskoboinik et al. 2001;

357 Valverde et al. 2008).

Like ATP7A, we also observed domain-specific alterations of CTR1 in schizophrenia.

359 Specifically, the transmembrane and intracellular segment of CTR1 was decreased, but not the 
extracellular portion (Figure 4B). CTR1, like ATP7A, is a transmembrane protein. The extracellular and intracellular portions both possess copper-binding domains (Eisses and Kaplan 2005); knockout of either region impairs copper uptake, though $<40 \%$ of uptake remains functional (Eisses and Kaplan 2005). Our transmembrane protein analysis includes the extracellular pore/channel opening (Eisses and Kaplan 2005); deficits in this region may affect extracellular copper binding and its transport to the cytosol, potentially resulting in decreased copper transport into the cell. The mechanism behind domain-specific alterations of CTR1 in schizophrenia remains untested. However, perhaps CTR1 transcription regulation is

368 pathologically altered. In yeast, CTRl contains a copper response element (CuRE) in its

369 promoter, targeted by transcription factor Mac1, which regulates CTR1 protein expression in a 370 copper-dependent manner (Labbe et al. 1997). During times of copper starvation, CTRl should

371 be upregulated. However, single or multiple point mutations within the CuREs suppress both

372 copper-dependent repression and expression of CTRl (Labbe et al. 1997). Given the plethora of 373 genetic alterations in schizophrenia, perhaps CTR1 is yet another to add to the list. Additionally,

374 CTR1 may contribute to the genetic alterations in schizophrenia, as it is required for Ras and

375 MAPK signaling (Turski et al. 2012; Tsai et al. 2012). Further study is needed to determine 376 genetic regulation of CTRl expression in human cells and its implications for schizophrenia.

377 Given the decreased CTR1, buildup of copper in the plasma of schizophrenia subjects

378 (Vidovic et al. 2013), and the dysregulation of kinases and phosphorylation in schizophrenia

379 (Banerjee et al. 2015; Sing 2013; Shelton et al. 2015; Ramkumar et al. 2018), we suggest a

380 potential alteration of the copper-sensing system of the metal binding domains and the cascading

381 system of phosphorylation thereafter in unmedicated schizophrenia that is potentially corrected

382 with antipsychotic treatment. Our finding of increased C-terminal ATP7A in medicated subjects 
suggests further efforts by the cell to restore copper homeostasis. ATP7A is constantly recycled between the plasma membrane and the TGN, but maintains a steady-state localization in the TGN during normal copper conditions (Yamaguchi et al. 1993). The carboxy terminus of the protein is responsible for endocytosis from the plasma membrane to the TGN (Petris et al. 1996;

387 Petris and Mercer 1999; Francis et al. 1999). By increasing localization of ATP7A to the TGN,

388 relatively increased copper insertion into metalloenzymes and the secretory pathway should 389 occur.

In brain, ATP7B moves copper from the parenchyma through the blood brain barrier to

391 the blood (Figure 1A). ATP7B deficits result in Wilson's disease, characterized by high copper

392 accumulation in brain and liver and low accumulation in blood (Wilson 1934). It is therefore

393 possible that an increase in ATP7B could be causing the increases in blood copper levels often

394 reported in schizophrenia. We did not observe any alterations of the copper transporter ATP7B, 395 narrowing down the possible mechanisms by which increased blood copper in schizophrenia 396 could occur.

398 Implications

Given that ATP7A, dysbindin and CTR1 are located, in part, in the astrocytic end feet

400 that form the BBB (Scheiber et al. 2010; Iijima et al. 2009; Nyguyen et al. 2017) our results 401 suggest abnormalities of the BBB in schizophrenia subjects. If less copper is transported across 402 the BBB in schizophrenia, excess blood copper and a brain copper deficit could result. These 403 results could explain the conundrum of elevated copper levels in the blood of patients (see 404 Vidovic et al. 2013 and references therein), and the pathologies and behaviors reminiscent of 405 schizophrenia that occur during cellular copper starvation (Gokhale et al. 2015; Gregg et al. 
2009; Herring and Konradi 2011). In mice, copper starvation results in demyelination, impaired prefrontal cortex function, and schizophrenia-like behaviors such as deficits in novel object recognition, spatial memory tasks, pre-pulse inhibition, social interaction, and anxiety (Talbot et al. 2009; Gregg et al. 2009; Herring and Konradi 2011). Therefore, brain copper starvation may be an underlying contributor to the cognitive symptoms of schizophrenia.

Conclusions

The current study provides the first evidence of abnormal brain copper homeostasis in

414 schizophrenia. Our findings suggest protein-region specific abnormalities in copper transport and

415 isoform-specific dysbindin alterations. Intracellular copper binding appears to be decreased

416 while ATP7A endocytosis mediation is increased, but only the binding is modulated by

417 antipsychotic treatment. Our results suggest extracellular copper binding and transport into the

418 cell via CTR1 is impaired in schizophrenia, and not rescued with treatment. Our findings not

419 only provide information on a potentially new pathological mechanism, but may provide a link

420 between several well-known but seemingly segregated findings in schizophrenia. Lastly, these

421 results may elucidate the paradox of excess copper in schizophrenia blood and the schizophrenia-

422 like pathology that occurs in a copper-deficit state. However, schizophrenia is a complex

423 disorder and therefore copper alterations as a contributing pathology in schizophrenia merit

424 further study. Further elucidation could provide vital information about the cellular pathology of

425 schizophrenia and open new avenues for treatment. 
429 study. Additionally, this work was supported by the National Institute of Mental Health

430 R0166123 and R21MH108867 to R.C.R, as well as the National Institute of Neurological

431 Disorders and Stroke F99NS105208 to K.E.S.

432

433 Disclosure of Interest

434 The authors have no competing interests and no disclosures. 
Table 1. (1A) Demographics and other information; mean and standard deviation. Some parameter information was not applicable for NCs. Therefore, an ANOVA was performed for parameters comparing three groups, and a t-test performed for parameters containing two groups. (1B) Correlational analyses of demographic and tissue quality markers. Comparisons between all proteins and demographic variables were made; significant p-values only are shown (bold). Only the relationship between dysbindin $1 \mathrm{~B} / \mathrm{C}$ and years frozen was significantly different between controls and schizophrenia subjects. While significant comparisons of coefficients were observed between medicated subjects for dysbindin $1 \mathrm{~B} / \mathrm{C}$ and postmortem interval, this demographic variable was not used as a covariate because no influence of medication status was found for dysbindin 1B/C. Abbreviations: Normal controls (NC); schizophrenia subjects (SZ); schizophrenia subject on medication (SZ-ON); schizophrenia subject off medication (SZ-OFF); postmortem interval (PMI); years sample has been frozen (YF); duration of illness (DUI); whole sample (WS); comparison of coefficients (CC); Caucasian (C); African American (AA).

Figure 1. Schematic of copper transport between the blood and brain in controls (A) and schizophrenia (B). Stars show copper. CTR1, white arrows; ATP7A, black arrows; ATP7B, striped arrows; BBB, blood brain barrier; BCB, blood cerebrospinal barrier. Thinner arrows indicate decreased protein levels.

Figure 2. (A) Representative western blots. Arrows indicate the primary band at the expected molecular weight, with the exception of C-terminus ATP7A. Full blots with detailed molecular weight markers are shown in supplementary figure 1 . Actin was a loading control. N-terminal ATP7A samples were not boiled; therefore non-boiled actin was the loading control. (B) Preadsorption blots for the proteins of interest. Arrows indicate the band that was measured and successfully blocked in two different normal controls (S1, S2). Control blots that incubated with primary antibody and no blocking peptide are shown on the left (control); preadsorbed blots are shown on the right (blocking peptide). MWM, molecular weight marker.

Figure 3. Protein levels of ATP7A (A-B), CTR1 (C-D), dysbindin 1A, 1B/C (E-F) and ATP7B $(\mathrm{G}-\mathrm{H})$ for analysis of diagnostic group and treatment status. Error bars represent standard deviation. Significant omnibus ANOVA results are: N-terminal ATP7A: $p=0.02$; C-terminal ATP7A: $p=0.01$; transmembrane CTR1: $p=0.001$. Significant ANOVAs were followed by post hoc tests illustrated by the following: *: $\mathrm{p}<0.05 ; * *: \mathrm{p}<0.01 ; * * *: \mathrm{p}<0.001$.

Figure 4. Numerical labeling of amino acid ranges are shown for each protein segment. Dotted boxes indicate antibody-specific epitopes. A. ATP7A protein structure. N-terminal antibody specifically binds to 225-273aa; C-terminal antibody binds to 1403-1452aa. B. CTR1 protein structure. Extracellular CTR1 antibody binds to 19-68aa; transmembrane CTR1 antibody specifically binds to 140-190aa. C. Intracellular diagram of copper chaperones and enzymes and schizophrenia-related alterations. Copper enters neurons after transport from astrocytes via CTR1 and is immediately bound by MT/GSH. MT/GSH then delivers copper to chaperone ATOX1 or the TGN where it is delivered to other metalloenzymes (e.g., SCO1 $\rightarrow$ COX) via ATP7A. The SN exhibits more ATOX1 than other brain regions. SCO1 is a copper-requiring enzyme involved in the last step of the electron transport chain of ATP synthesis. SCO1, 
481 MT/GSH, COX, ATP, ATP7A and CTR1 are all downregulated or altered in schizophrenia. 482 Abbreviations: MT, metallothionein; GSH, glutathione; APD, antipsychotic drug; SN, substantia 483 nigra; COX, cytochrome c oxidase. References: 1) The present paper; 2) Scheiber et al. 2010; 3) 484 Maryon et al. 2013; 4) Do et al. 2000; 5) Xuan et al. 2015; 6) Davies et al. 2013; Prohaska 1987; 485 7) Petris et al. 1996; 8) Leary et al. 2007; 9) Purcell et al. 2014; 10) Cavelier et al. 1995; and 11) 486 Volz et al. 2000. 
Banerjee A, Wang HY, Borgmann-Winter KE, Macdonald ML, Kaprielian H, Stucky A, et al. 2015. Src kinase as a mediator of convergent molecular abnormalities leading to NMDAR hypoactivity in schizophrenia. Mol psychiatry 20(9) 1091-100.

Burdick KE, Lencz T, Funke B, Finn CT, Szeszko PR, Kane JM. 2006. Genetic variation in DTNBP1 influences general cognitive ability. Hum Mol Gen. 15: 1563-1568.

Carr GV, Jenkins KA, Weinberger DR. Papaleo F. 2013. Loss of dysbindin-1 in mice impairs reward-

497 Cavelier L, Jazin EE, Eriksson I, et al. 1995. Decreased cytochrome-c oxidase activity and lack of agerelated accumulation of mitochondrial DNA deletions in the brains of schizophrenics. Genomics. 29: 217499224.

Cox MM, Tucker AM, Tang J, et al. 2009. Neurobehavioral abnormalities in the dysbindin-1 mutant, sandy, on a C57BL/6J genetic background. Genes Brain Behav. 8: 390-397.

502 Davies KM, Hare DJ, Cottam V, et al. 2013. Localization of copper and copper transporters in the human 503 brain. Metallomics. 5: 43-51.

504 Dickman DK, Davis GW. 2009. The schizophrenia susceptibility gene dysbindin controls synaptic 505 homeostasis. Science. 326: 1127-1130.

Eisses JF, Kaplan JH. 2005. The mechanism of copper uptake mediated by human CTR1: a mutational analysis. J Biol Chem. 280:37159-37168.

510 Faludi G, Mirnics K. 2011. Synaptic changes in the brain of subjects with schizophrenia. Int J Dev 511 Neurosci. 29:305-309.

Francis MJ, Jones EE, Levy ER, Martin RL, Ponnambalam S, Monaco AP. 1999. Identification of a dileucine motif within the $\mathrm{C}$ terminus domain of the Mnekes disease protein that mediates endocytosis from

514 the plasma membrane. J Cell Sci. 112:1721-1732.

515 GeneCard, 2016. Genecards.org. Search ATP7A human.

516 Gokhale A, Vrailas-Mortimer A, Larimore J, et al. 2015. Neuronal copper homeostasis susceptibility by 517 genetic defects in dysbindin, a schizophrenia susceptibility factor. Hum Mol Genet. 24: 5512-5523.

518 Gregg JR, Herring NR, Naydenov AV, Hanlin RP, Konradi C. 2009. Downregulation of oligodendrocyte 519 transcripts is associated with impaired prefrontal cortex function in rats. Schizophr Res. 113: 277-287.

520 Halliwell B, Gutteridge JM. 2007. Free Radicals in Biology and Medicine. Oxford University Press: New 521 York, NY. 
Herring NR, Konradi C. 2011. Myelin, copper, and the cuprizone model of schizophrenia. Front Biosci (Schol Ed). 3: 23-40.

524 Howes O, Williams M, Ibrahim K, et al. 2013. Midbrain dopamine function in schizophrenia and 525 depression: a post-mortem and positron emission tomographic imaging study. Brain. 136: 3242-3251.

526 Hua H, Georgiev O, Schaffner W, Steiger D. 2010. Human copper transporter CTR1 is functional in drosophila, revealing a high degree of conservation between mammals and insects. J Biol Inorg Chem. 15:107-13.

Iijima S, Masaki H, Wakayama Y, et al. 2009. Immunohistochemical detection of dysbindin at the astroglial endfeet around the capillaries of mouse brain. J Mol Hist. 40: 117-121.

531 Labbe S, Zhu Z, Thiele DJ. 1997. Copper-specific transcriptional repression of yeast genes encoding 532 critical components in the copper transport pathway. J Biol Chem. 272: 15951-8.

533 Leary SC, Cobine PA, Kaufman BA, et al. 2007. The human cytochrome c oxidase assembly factors 534 SCO1 and SCO2 have regulatory roles in the maintenance of cellular copper homeostasis. Cell Metab. 5: 535 9-20.

536 Lee J, Prohaska JR, Thiele DJ. 2001. Essential role for mammalian copper transporter Ctrl in copper 537 homeostasis and embryonic development. Proc Natl Acad Sci USA. 12: 6842-6847.

538 Lutsenko S, Barnes NL, Bartee MY, Dmitriev OY. 2007. Function and regulation of human copper539 transporting ATPases. Physiological Reviews 87(3), 1011-1046.

540 Maryon EB, Molloy SA, Kaplan JH. 2013. Cellular glutathione plays a key role in copper uptake 541 mediated by human copper transporter 1. Am J Physiol Cell Physiol. 304: C768-779.

542 Menkes JH, Alter M, Steigleder GK, Weakly DR, Sung JH. 1962. A sex-linked recessive disorder with 543 retardation of growth, peculiar hair, and focal cerebral and cerebellar degeneration. Pediatrics. 29: 764544779.

545 Schoonover K, Nguyen C, Farmer C, Roberts RC. 2018. CTR1 in normal striatum, substantia nigra, and 546 cortex. Poster presented at Society for Neuroscience, San Diego, California.

547 Nickl-Jockschat T, Stöcker T, Markov V, et al. 2012. The impact of a Dysbindin schizophrenia 548 susceptibility variant on fiber tract integrity in healthy individuals: a TBSS-based diffusion tensor 549 imaging study. Neuroimage. 60: 847-853.

550 Numakawa T, Yagasaki Y, Ishimoto T, et al. 2004. Evidence of novel neuronal function of dysbindin, a 551 susceptibility gene for schizophrenia. Hum Mol Genet. 13:2699-2708.

552 Papaleo F, Yang F, Garcia S, et al. 2012. Dysbindin-1 modulates prefrontal cortical actibity and 553 schizophrenia-like behaviors via dopamine/D2 pathways. Mol Psychiatry. 17:85-98.

554 Parent M, Parent, A, Charara A. 1999. Glutamatergic inputs to midbrain dopaminergic neurons in 555 primates. Parkinsonism Relat Disord. 5:193-201. 
Petris MJ, Mercer JF, Culvenor JG, Lockhart P, Gleeson PA, Camakaris J. 1996. Ligand-regulated transport of the Menkes copper P-type ATPase efflux pump from the Golgi apparatus to the plasma membrane: a novel mechanism of regulated trafficking. EMBO J. 15: 6084-6095.

560 Petris MJ, Mercer JF. 1999. The Menkes protein (ATP7A; MNK) cycles via the plasma membrane in 561 both basal and elevated extracellular copper using a C-terminal di-leucine endocytic signal. Hum Mol

562 Genet. 8:2107-2115.

563 Prohaska JR. 1987. Functions of trace elements in brain metabolism. Physiol Rev. 67: 858-901.

564 Purcell SM, Moran JL, Fromer M, et al. 2014. A polygenic burden of rare disruptive mutations in 565 schizophrenia. Nature. 506: 185-190.

566 Ramkumar A, Jong BY, Ori-McKenney KM. 2018. ReMAPping the microtubule landscape: how 567 phosphorylation dictates the activities of microtubule associated proteins. Dev Dyn 247(1), 138-155.

Roberts RC, Roche JK, Somerville SM, Conley RR. 2012. Ultrastructural distinctions between treatment responders and non-responders in schizophrenia: postmortem studies of the striatum. In Labate L (ed). Mental Illnesses - Evaluation, Treatments, and Implications. InTech: Croatia. 261-286.

571 Sato M, Ohtomo K, Daimon T, Sugiyama T, Iijima K. 1994. Localization of copper to afferent terminals 572 in rat locus ceruleus, in contrast to mitochondrial copper in cerebellum. J Histochem Cytochem. 42: 15855731591.

574 Scheiber IF, Mercer JF, Dringen R. 2010. Copper accumulation by cultured astrocytes. Neurochem Int. 575 56: 451-460. 108 schizophrenia-associated genetic loci. Nature. 511:421-427.

Schoonover KE, Roberts RC. 2016. Abnormalities in the copper transporters ATP7A and CTR1 in postmortem substantia nigra in schizophrenia. Annual meeting for the American College of

580 Neuropsychopharmacology.

Schoonover KE, McCollum LA, Roberts RC. 2017. Protein markers of neurotransmitter synthesis and

582 release in postmortem schizophrenia substantia nigra. Neuropyschopharmacology. 42:540-550.

Schwyn RC and Fox CA. 1974. The primate substantia nigra: a Golgi and electron microscopic study, J Hirnforsch. 15: 95.

Singh KK. 2013. An emerging role for Wnt and GSK3 signaling pathways in schizophrenia.

587 Developmental Biology: Frontiers for Clinical Genetics. 
Stan AD, Ghose S, Gao XM, et al. 2006. Human postmortem tissue: what quality markers matter? Brain Res. 1123: 1-11.

Straub RE, Jiang Y, MacLean CJ, et al. 2002. Genetic variation in the 6p22.3 gene DTNBP1, the human ortholog of the mouse dysbindin gene, is associated with schizophrenia. Am J Hum Genet. 71: 337-348.

Talbot K. 2009. The sandy (sdy) mouse: a dysbindin-1 mutant relevant to schizophrenia research. Prog

Talbot K, Eidem WL, Tinsley CL, Benson MA, Thompson EW, Smith RJ. 2004. Dysbindin-1 is reduced in intrinsic, glutamatergic terminals of the hippocampal formation in schizophrenia. Journal of Clinical Investigation. 113: 1353-1363.

Talbot K, Louneva N, Cohen JW, Kazi H, Blake DJ, Arnold SE. 2011. Synaptic dysbindin-1 reductions in schizophrenia occur in an isoform-specific manner indicating their synaptic location. PLOS One. 6:

604 e16886.

Talbot K, Ong WY, Blake DJ, et al. 2009. Dysbindin-1 and its protein family with special attention to the potential role of dysbindin-1 in neuronal functions and the pathophysiology of schizophrenia. In D. Javitt \& J. Kantorowitz (Eds.), Handbook of Neurochemistry and Molecular Neurobiology (3rd ed., Vol. 27). Springer, New York, 107-241.

Tang J, LeGros RP, Louneva N, et al. 2009. Dysbindin-1 in dorsolateral prefrontal cortex of schizophrenia cases is reduced in an isoform-specific manner unrelated to dysbindin-1 mRNA expression. Hum Mol Genet. 18: 3851-3863.

Tepper JM, Lee CR. 2007. GABAergic control of substantia nigra dopaminergic neurons. Prog brain Res. 160:189-208.

617 Tsai CY, Finley JC, Ali SS, Patel HH, Howell SB. 2012. Copper influx transporter 1 is required for FGF, 618 PDGF, and EGF-induced MAPK signaling. Biochem Pharmacol. 84:1007-13.

619 Turski ML, Brady DC, Kim HJ, Kim BE, Nose Y, Counter CM, et al. 2012. A novel role for copper in 620 Ras/mitogen-activated protein kinase signaling. Mol Cell Biol. 32: 1284-95.

621 Valverde RHF, Morin I, Lowe J, Mintz E, Cuillel M, Vieyra A. 2008. Cyclic AMP-dependent protein 622 kinase controls energy interconversion during the catalytic cycle of the yeast copper-ATPase. FEBS 623 Letters 582(6), 891-895.

624 Varela-Gomez N, Mata I, Perez-Iglesias R, et al. 2015. Dysbindin gene variability is associated with 625 cognitive abnormalities in first-episode non-affective psychosis. Cognitive Neuropsychiatry. 20: 144-156. 
626 Vidovic B, Dordevic B, Milovanovic S, et al. 2013. Selenium, zinc, and copper plasma levels in patients with schizophrenia: Relationship with metabolic risk factors. Biol Trace Elem Res. 156: 22-28.

628 Volz HR, Riehemann S, Maurer I, et al. 2000. Reduced phosphodiesters and high-energy phosphates in 629 the frontal lobe of schizophrenic patients: a (31)P chemical shift spectroscopic-imaging study. Biol

630 Psychiatry. 47: 954-961.

631 Voskoboinik I, Mar J, Strausak D, Camakaris J. 2001. The regulation of catalytic activity of the menkes 632 copper translocating P-type ATPase. Role of high affinity copper-binding sites. J Biol Chem 276(30), $63328620-7$.

634 Weickert CS, Rothmond DA, Hyde TM, Kleinman JE, Straub RE. 2008. Reduced DTNBP1 (dysbindin-1) 635 mRNA in the hippocampal formation of schizophrenia patients. Schizophr Res. 98: 105-110.

636 Weickert CS, Straub RE, McClintock BW, et al. 2004. Human dysbindin (DTNBP1) gene expression in 637 normal brain and in schizophrenic prefrontal cortex and midbrain. Arch Gen Psychiatry. 61: 544-555.

638 Wilson SA. 1934. Kayser-Fleischer ring in cornea in two cases of Wilson's disease (Progressive 639 Lenticular Degeneration). Proc R Soc Med. 27: 297-298.

640 Xiao L, Xu H, Zhang Y, et al. 2008. Quetiapine facilitates oligodendrocyte development and 641 prevents mice from myelin breakdown and behavioral changes. Mol Psychiatry. 13:697-708.

642 Xuan Y, Yan G, Wu R, Huang Q, Li X, Xu H. 2015. The cuprizone-induced changes in (1)H-MRS 643 metabolites and oxidative parameters in C57BL/6 mouse brain: effects of quetiapine. Neurochem Int. 90:

$644 \quad 185-192$.

645 Yamaguchi Y, Heiny ME, Suzuki M, Gitlin JD. 1996. Biochemical characterization and intracellular 646 localization of the Menkes disease protein. Proc Natl Acad Sci USA. 93: 14030-14035.

647 Yamaguchi Y, Heiny ME, Gitlin JD. 1993. Isolation and characterization of a human liver cDNA as a 648 candidate gene for Wilson disease. Biochemical and Biophysical research communications. 197: 271-77. 
Table 1A. Demographics and Markers of Tissue Quality

\begin{tabular}{c|c|c|c|c|c|c|c|c|c}
\hline & $\#$ & Age, years & $\begin{array}{c}\text { PMI, } \\
\text { hours }\end{array}$ & pH & YF & Age of Onset & DUI, years & Race & Sex \\
\hline NC & 11 & $50.9 \pm 16.3$ & $15.6 \pm 7.1$ & $6.7 \pm 0.2$ & $17.4 \pm 4.7$ & N/A & N/A & $8 \mathrm{C} / 3 \mathrm{AA}$ & $9 \mathrm{M} / 2 \mathrm{~F}$ \\
\hline $\mathrm{SZ}$ & 15 & $42.1 \pm 12.2$ & $13.6 \pm 8.9$ & $6.5 \pm 0.4$ & $19.3 \pm 3.0$ & $22.6 \pm 5.1$ & $18.6 \pm 9.3$ & $9 \mathrm{C} / 6 \mathrm{AA}$ & $11 \mathrm{M} / 4$ \\
\hline t-test & & $\mathrm{p}=0.13$ & $\mathrm{p}=0.55$ & $\mathrm{p}=0.30$ & $\mathrm{p}=0.22$ & N/A & N/A & $\chi^{2}=0.50$ & $\boldsymbol{\chi}^{2}=0.6$ \\
\hline SZ-On & 11 & $50.9 \pm 16.3$ & $15.2 \pm 9.3$ & $6.6 \pm 0.3$ & $18.1 \pm 2.6$ & $21.3 \pm 4.8$ & $19.9 \pm 9.4$ & $6 \mathrm{C} / 5 \mathrm{AA}$ & $7 \mathrm{M} / 4 \mathrm{~F}$ \\
\hline SZ-Off & 4 & $50.3 \pm 17.5$ & $11.0 \pm 8.6$ & $6.4 \pm 0.4$ & $21.8 \pm 3.0$ & $27.0 \pm 4.2$ & $14.0 \pm 9.9$ & $1 \mathrm{C} / 3 \mathrm{AA}$ & $4 \mathrm{M} / 0 \mathrm{~F}$ \\
\hline $\begin{array}{c}\text { ANOVA/ } \\
\text { t-test }\end{array}$ & $\mathrm{p}=0.13$ & $\mathrm{p}=0.45$ & $\mathrm{p}=0.60$ & $\mathrm{p}=0.15$ & $\mathrm{p}=0.18$ & $\mathrm{p}=0.46$ & $\chi^{2}=0.20$ & $\boldsymbol{\chi}^{\mathbf{2}}=0.1$ \\
\hline
\end{tabular}

\begin{tabular}{|c|c|c|c|c|c|}
\hline \multicolumn{6}{|c|}{ Table 1B. Demographic/Protein Correlations } \\
\hline & $\begin{array}{c}\text { N-ATP7A/ } \\
\text { Age }\end{array}$ & $\begin{array}{l}\text { ATP7B/ } \\
\text { Age }\end{array}$ & $\begin{array}{c}\text { T-CTR1/ } \\
\text { YF }\end{array}$ & $\begin{array}{c}\text { Dys 1BC/ } \\
\text { YF }\end{array}$ & $\begin{array}{c}\text { Dys 1BC/ } \\
\text { PMI }\end{array}$ \\
\hline WS & $\begin{array}{c}\mathrm{p}=0.09 \\
\mathrm{r}=-0.34\end{array}$ & $\begin{array}{c}p=0.04 \\
r=-0.41\end{array}$ & $\begin{array}{c}p=0.03 \\
r=-0.43\end{array}$ & $\begin{array}{c}\mathrm{p}=0.10 \\
\mathrm{r}=-0.34\end{array}$ & $\begin{array}{c}\mathrm{p}=0.39 \\
\mathrm{r}=-0.18\end{array}$ \\
\hline $\mathbf{N C}$ & $\begin{array}{c}p=0.04 \\
r=-0.62\end{array}$ & $\begin{array}{l}\mathrm{p}=0.19 \\
\mathrm{r}=-0.43\end{array}$ & $\begin{array}{c}\mathrm{p}=0.17 \\
\mathrm{r}=-0.44\end{array}$ & $\begin{array}{c}p=0.02 \\
r=-0.69\end{array}$ & $\begin{array}{l}p=0.73 \\
r=0.12\end{array}$ \\
\hline SZ & $\begin{array}{c}\mathrm{p}=0.51 \\
\mathrm{r}=-0.19\end{array}$ & $\begin{array}{c}\mathrm{p}=0.77 \\
\mathrm{r}=-0.08\end{array}$ & $\begin{array}{l}\mathrm{p}=0.35 \\
\mathrm{r}=-0.26\end{array}$ & $\begin{array}{l}\mathrm{p}=0.62 \\
\mathrm{r}=0.14\end{array}$ & $\begin{array}{l}\mathrm{p}=0.39 \\
\mathrm{r}=0.24\end{array}$ \\
\hline $\begin{array}{c}\mathrm{CC} \\
\mathrm{NC} \text { vs SZ }\end{array}$ & $\mathrm{p}=0.23$ & $\mathrm{p}=0.41$ & $\mathrm{p}=0.64$ & $\mathbf{p}=\mathbf{0 . 0 3}$ & $\mathrm{p}=0.79$ \\
\hline OFF & $\begin{array}{l}\mathrm{p}=0.97 \\
\mathrm{r}=0.03\end{array}$ & $\begin{array}{c}\mathrm{p}=0.27 \\
\mathrm{r}=-0.73\end{array}$ & $\begin{array}{c}\mathrm{p}=0.21 \\
\mathrm{r}=-0.79\end{array}$ & $\begin{array}{c}\mathrm{p}=0.71 \\
\mathrm{r}=-0.29\end{array}$ & $\begin{array}{c}p=0.03 \\
r=-0.97\end{array}$ \\
\hline $\mathbf{O N}$ & $\begin{array}{c}\mathrm{p}=0.92 \\
\mathrm{r}=-0.04\end{array}$ & $\begin{array}{c}\mathrm{p}=0.16 \\
\mathrm{r}=-0.46\end{array}$ & $\begin{array}{l}\mathrm{p}=0.82 \\
\mathrm{r}=0.08\end{array}$ & $\begin{array}{l}\mathrm{p}=0.38 \\
\mathrm{r}=0.30\end{array}$ & $\begin{array}{l}\mathrm{p}=0.45 \\
\mathrm{r}=0.26\end{array}$ \\
\hline $\begin{array}{c}\text { CC } \\
\text { OFF vs ON }\end{array}$ & $\mathrm{p}=0.94$ & $\mathrm{p}=0.68$ & $\mathrm{p}=0.14$ & $\mathrm{p}=0.57$ & $p=0.03$ \\
\hline $\begin{array}{c}\text { CC } \\
\text { OFF vs NC }\end{array}$ & $\mathrm{p}=0.47$ & $\mathrm{p}=0.66$ & $\mathrm{p}=0.57$ & $\mathrm{p}=0.24$ & $p=0.04$ \\
\hline $\begin{array}{c}\text { CC } \\
\text { ON vs NC }\end{array}$ & $\mathrm{p}=0.16$ & $\mathrm{p}=0.94$ & $\mathrm{p}=0.25$ & $p=0.02$ & $\mathrm{p}=0.77$ \\
\hline
\end{tabular}


A. Transcellular Transport

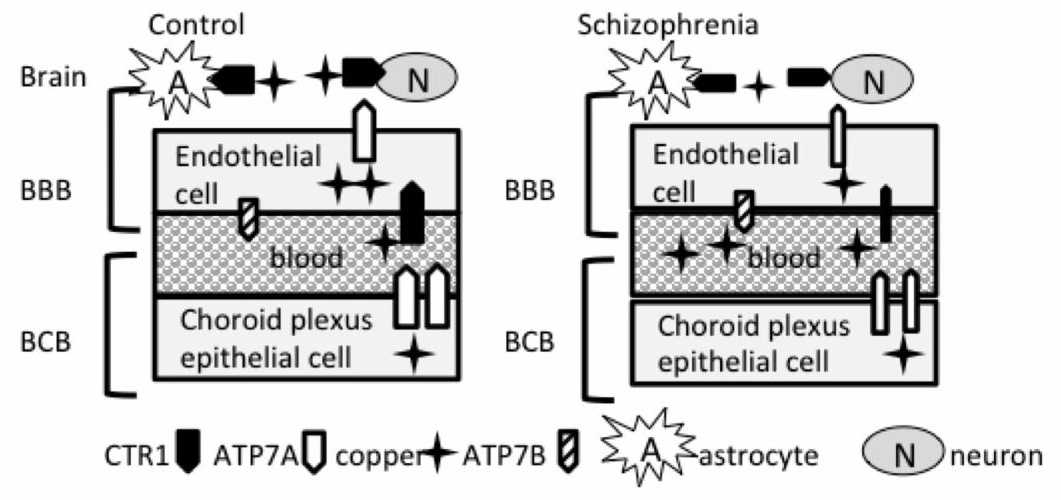

B. Intracellular Transport

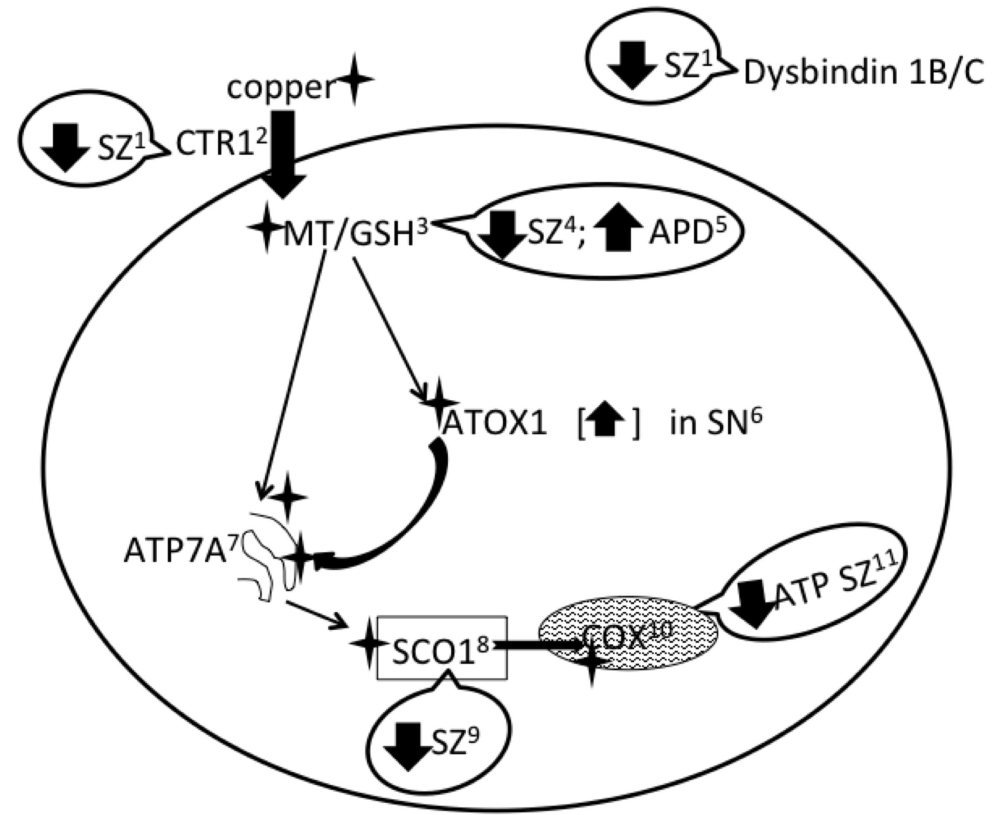


A

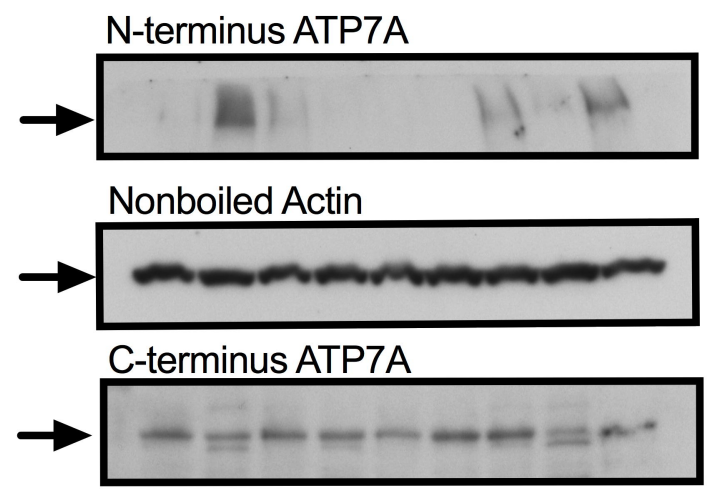

Extracellular CTR1

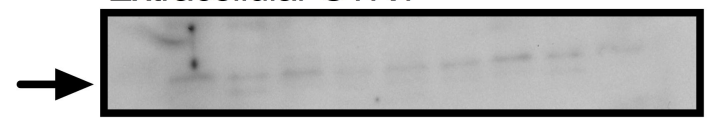

Transmembrane CTR1

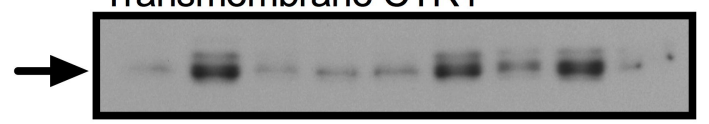

Dysbindin $1 \mathrm{~A}$

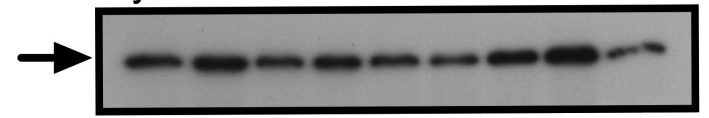

Dysbindin 1B/C
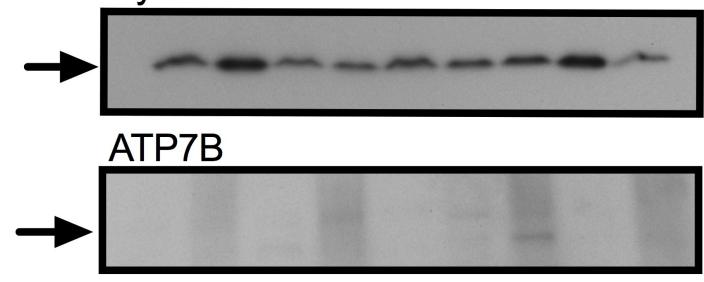

Boiled Actin

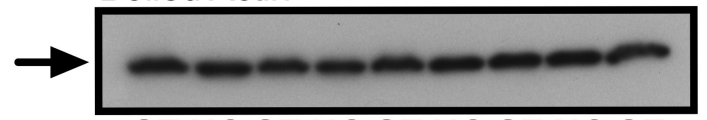

SZ NC SZ NC SZ NC SZ NC SZ OFF ON ON ON ON $\begin{array}{lllll}\text { OFF } & R & R & T R & \text { TR }\end{array}$
$\mathrm{N}$-terminus ATP7A

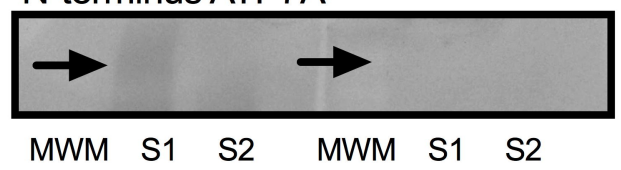

C-terminus ATP7A

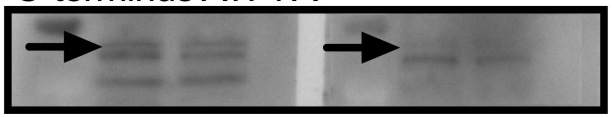

\section{Extracellular CTR1}

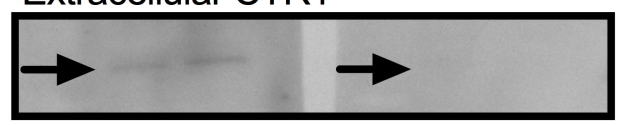

Transmembrane CTR1

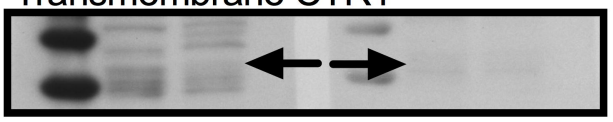

MWM S1 S2 $\quad$ MWM S1 S2 
A

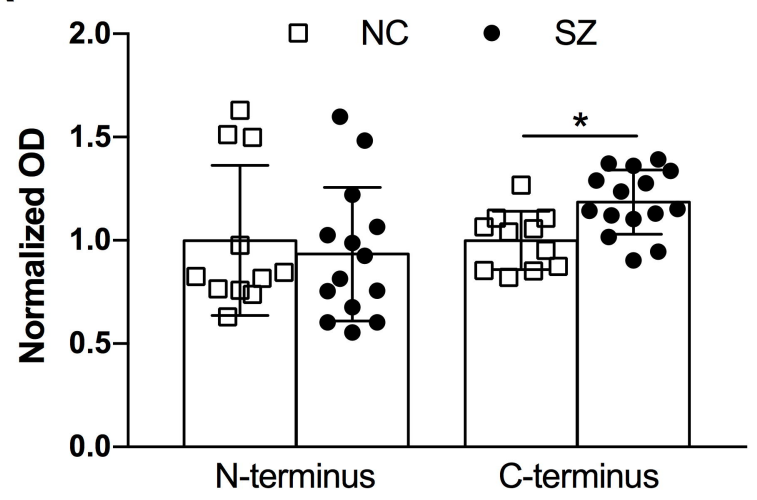

C

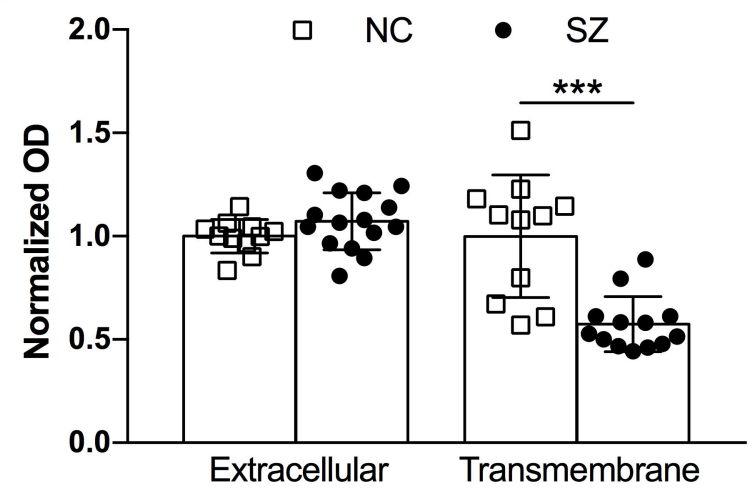

E

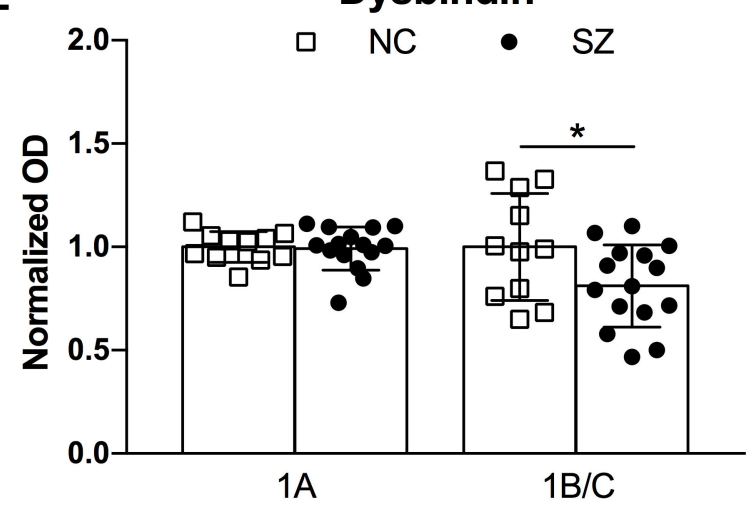

G

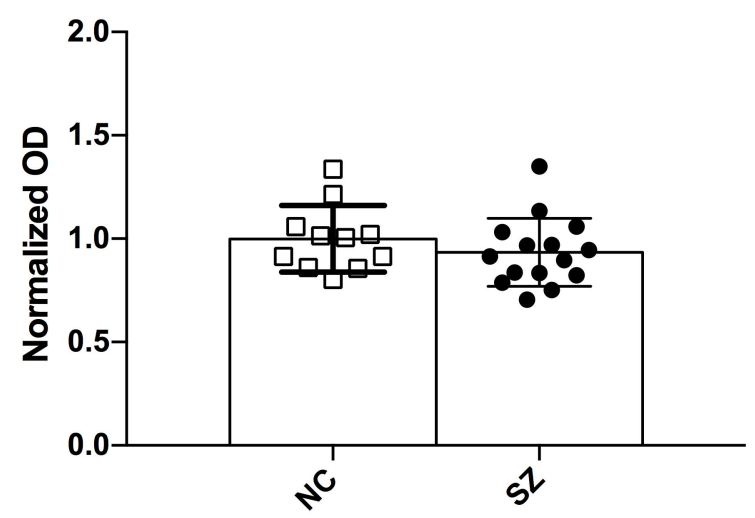

B

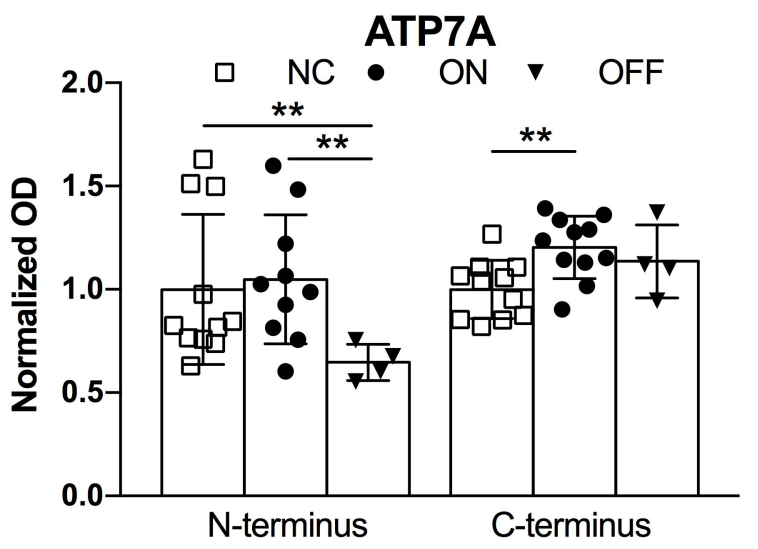

D

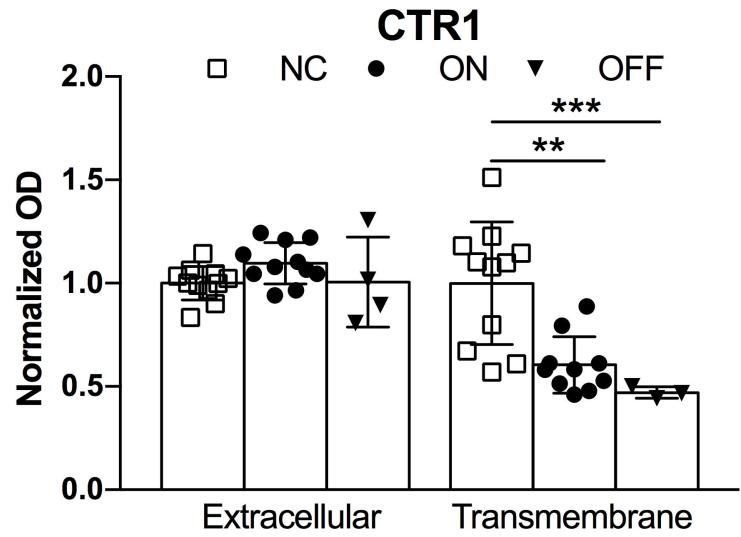

F

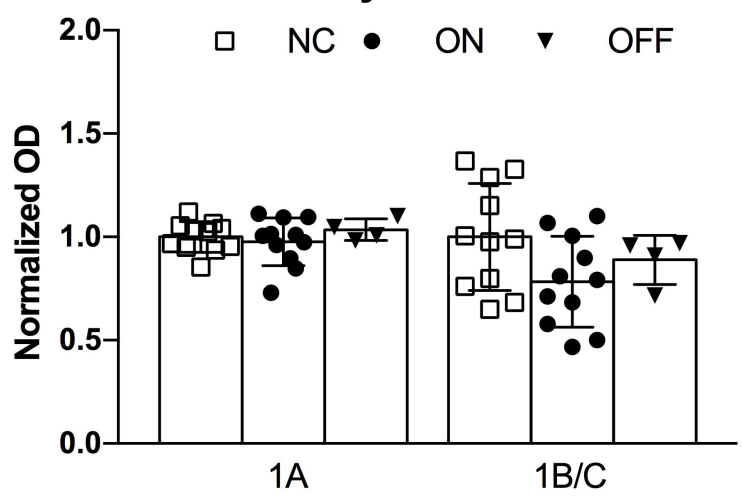

H

ATP7B

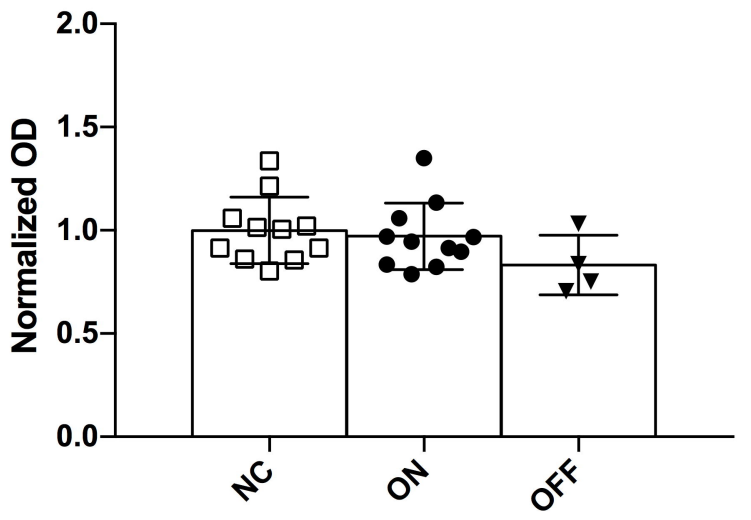




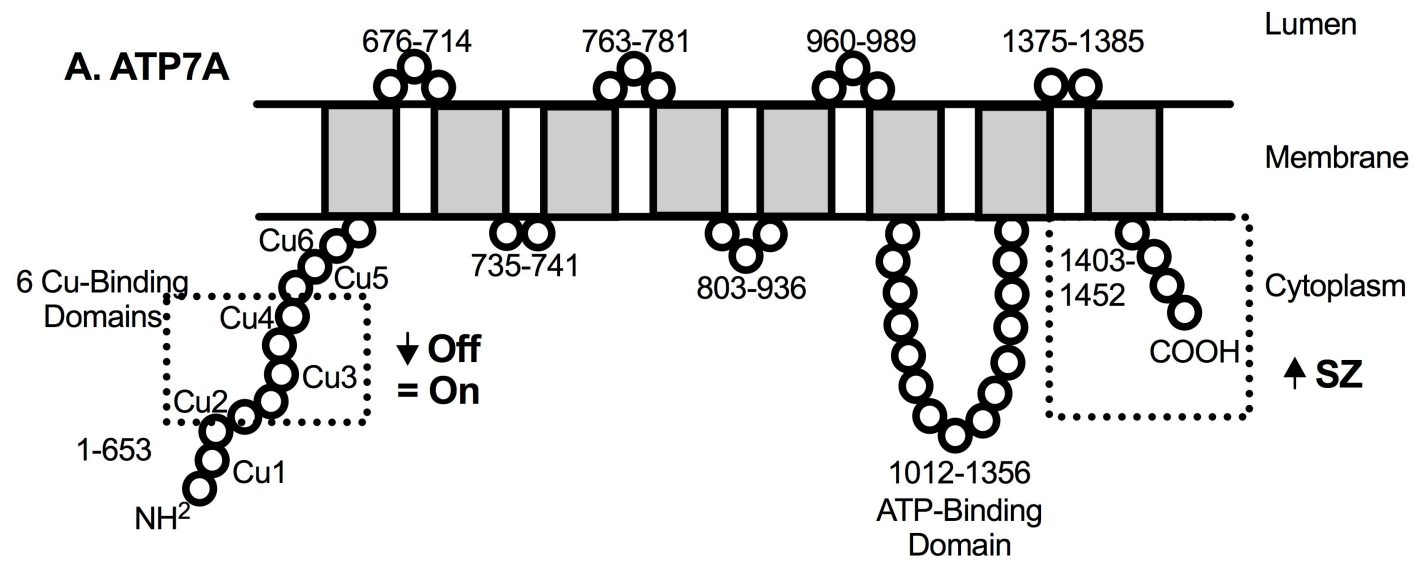

\section{B. CTR1}

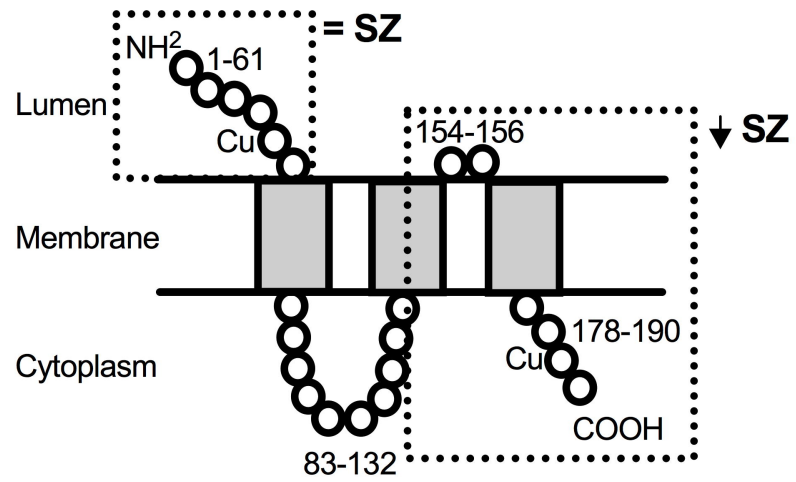

